\title{
Criminologie
}

\section{La prédiction des contacts avec le tribunal de la jeunesse : utilisation de l'évaluation du comportement par les pairs au début de l'école primaire}

\author{
Richard E. Tremblay, Lise Desmarais-Gervais, Marc LeBlanc et Alexandre \\ Schwartzman
}

Volume 19, numéro 2, 1986

La prédiction de la carrière criminelle

URI : https://id.erudit.org/iderudit/017239ar

DOI : https://doi.org/10.7202/017239ar

Aller au sommaire du numéro

Éditeur(s)

Les Presses de l'Université de Montréal

ISSN

0316-0041 (imprimé)

1492-1367 (numérique)

Découvrir la revue

Citer cet article

Tremblay, R. E., Desmarais-Gervais, L., LeBlanc, M. \& Schwartzman, A. (1986).

La prédiction des contacts avec le tribunal de la jeunesse : utilisation de

l'évaluation du comportement par les pairs au début de l'école primaire.

Criminologie, 19(2), 33-48. https://doi.org/10.7202/017239ar

\section{Résumé de l'article}

This study sets out to verify to what extent the evaluations of the behaviour of children by their peers in first year primary school make it possible to predict those who will eventually end up with an open record at the Juvenile Court. During their first year at school the children are classified according to the following categories : aggressive, socially withdrawn, aggressive-withdrawn, neither aggressive/nor withdrawn (contrast). A total of 992 subjects (493 girls and 499 boys) were evaluated at two different times during the study. The results indicate that the "aggressive-withdrawn" subjects, among the girls, are more likely to have an open record at the Court during the 7 or 8 years after having been evaluated in first year primary school. These findings may indicate that the girls whose behaviour is aberrant are more easily identified by their peers, given the fact that they generally have fewer problems of adaptation than the boys during their first year of school. 
LA PREDICTION DES CONTACTS AVEC LE TRIBUNAL DE LA JEUNESSE : UTILISATION DE L'ÉVALUATION DU COMPORTEMENT PAR LES PAIRS AU DÉBUT DE L'ÉCOLE PRIMAIRE'

Richard E. Tremblay* Lise Desmarais-Gervais** Marc LeBlanc*** Alexandre Schwartzman****

This study sets out to verify to what extent the evaluations of the behaviour of children by their peers in first year primary school make it possible to predict those who will eventually end up with an open record at the Juvenile Court. During their first year at school the children are classified according to the following categories : aggressive, socially withdrawn, aggressivewithdrawn, neither aggressive/nor withdrawn (contrast). A total of 992 subjects (493 girls and 499 boys) were evaluated at two different times during the study. The results indicate that the "aggressive-withdrawn" subjects, among the girls, are more likely to have an open record at the Court during the 7 or 8 years after having been evaluated in first year primary school. These findings may indicate that the girls whose behaviour is aberrant are more easily identified by their peers, given the fact that they generally have fewer problems of adaptation than the boys during their first year of school.

\section{INTRODUCTION}

$\mathrm{Au}$ cours des trois dernières décennies quelques études prospectives ont tenté de vérifier la capacité de prédire l'inadapta-

1. Cette recherche a été réalisée grâce à des subventions du Programme national de subvention au bien-être social (ministère de la Santé et des Services sociaux du Québec). Nous remercions : l'équipe "high risque" du Centre de recherche sur le dêveloppement humain de l'Université Concordia pour avoir mis à notre disposition l'information sur les prédicteurs; le Tribunal de la jeunesse de Montréal et de Longueuil pour la mise à notre disposition de leurs dossiers après avis favorable de la Commission d'accès à l'information.

* Professeur à l'École de psychoéducation de l'Université de Montréal.

* * Membre du Groupe de recherche interuniversitaire sur la prévention de l'inadaptation psychosociale, Université de Montréal.

*** Professeur titulaire à l'École de criminologie et chercheur associé au Centre international de criminologie comparée, Université de Montréal.

**** Chercheur au Centre de recherche sur le développement humain, Université Concordia. 
tion sociale par l'évaluation de prédicteurs au cours de l'enfance. Loeber et Dishion (1983), ainsi que Loeber et StouthamerLoeber (1985) ont publié des revues critiques d'études tentant de prédire les comportements antisociaux à l'adolescence et à l'âge adulte. Ces travaux font suite aux travaux importants de Glueck et Glueck $(1972,1950)$ qui, par analyse rétrospective, avaient mis en évidence des prédicteurs apparemment valides de la conduite délinquante à l'adolescence. Loeber et StouthamerLoeber (1985) ont identifié 17 études prédictives utilisant, au moins en partie, des sujets âgés de moins de 12 ans lors de l'évaluation des prédicteurs. Onze de ces études proviennent des États-Unis, quatre proviennent de pays scandinaves et deux d'Angleterre. À notre connaissance, aucune étude prédictive prospective des comportements antisociaux, par des prédicteurs à l'enfance, n'a été réalisée dans un pays francophone. Il est à remarquer également que la très grande majorité des études rapportées par Loeber et Stouthamer-Loeber portent sur des garçons ( 13 sur 17), alors que les 4 études incluant des filles ne distinguent pas garçons et filles.

Nous présentons ici les résultats d'une étude prédictive pour un échantillon de garçons et de filles francophones de Montréal, dont le comportement a été évalué alors qu'ils étaient en première année d'école primaire (6-7 ans). L'évaluation des cas officiels de délinquance et d'inadaptation sociale a été effectuée pour les sept ou huit années qui ont suivi.

$\grave{A}$ date, les comportements de l'enfant et l'environnement familial se sont avérés les meilleurs prédicteurs des difficultés d'adaptation sociale lorsque l'on utilise des sujets évalués dès l'enfance (voir Loeber et Stouthamer-Loeber, 1985). L'étude longitudinale de West et Farrington, 1977; Farrington, 1983; West, 1982 ainsi que celle de Craig et Glick (1968) sont d'excellents exemples. Dans la première étude réalisée sur un échantillon de garçons à Londres, les résultats montrent que les comportements "dérangeants» entre 8 et 10 ans (troublesome) en classe, tel qu'évalués par les pairs et les enseignants sont les meilleurs prédicteurs de la délinquance à 17 ans; la valeur de ce prédicteur est suivie de la criminalité chez les parents, les comportements parentaux inadéquats, le faible revenu familial, le nombre d'enfants dans la famille et le faible quotient intellectuel (West, 1982). L'étude de Craig et Glick (1968) par ailleurs, montre que l'évaluation de la qualité de l'environnement fami- 
lial (discipline et supervision de la mère ainsi que cohésion de l'unité familiale) ajoutée à celle du comportement par les enseignants en première année du primaire permet de prédire que $55 \%$ des garçons auront un dossier judiciaire 10 ans plus tard.

Pour réaliser l'évaluation par les pairs, dans ces études, l'on demande généralement aux enfants d'une classe de nommer ceux qui, parmi eux, présentent le plus souvent les comportements décrits sur une liste prédéterminée (voir Eron et al., 1971; West et Farrington, 1973). Dans certains cas, la popularité et le rejet par les pairs sont évalués (Roff et Sells, 1968). Ledingham, Schwartzman et leurs collaborateurs (Ledingham, 1981; Schwartzman et al., 1985) ont mis au point une façon de classifier les résultats d'une procédure d'évaluation par les pairs (le Peer Evaluation Inventory de Pekarik et al., 1976) susceptible d'améliorer notre prédiction des comportements déviants à l'adolescence et à l'âge adulte. À l'origine, cette procédure a été mise au point pour la prédiction de la schizophrénie. L'hypothèse étant que les enfants qui manifestent des comportements de retrait social accentués tout en manifestant des comportements agressifs accentués sont plus à risque de schizophrénie que les enfants qui ne sont que retirés socialement ou les enfants qui ne sont qu'agressifs (Ledingham, 1981). Pour choisir les enfants «agressifs-retirés» à risque de schizophrénie, Ledingham, Schwartzman et leurs collaborateurs ont retenu tous les enfants qui, sur l'échelle d'agressivité aussi bien que sur celle du retrait social du PEI, avaient un score au-delà du $75^{\mathrm{e}}$ percentile. Donc les enfants qui se situent parmi les $25 \%$ plus agressifs aussi bien que parmi les $25 \%$ plus retirés socialement.

Si l'on se fie aux travaux antérieurs utilisant des instruments d'évaluation du comportement par les pairs (e.g. Huesmann et al., 1984; West et Farrington, 1973) nous pouvons présumer que les individus qui ont un score élevé à l'échelle "agressivité» du PEI sont les plus à risque de comportements antisociaux à l'adolescence. Cependant, une publication récente de Milich et Landau (1984) permet de présumer que les enfants évalués «agressifs-retirés» sont également à risque de comportements antisociaux à l'adolescence. Milich et Landau ont en effet montré que chez des garçons de 5 à 7 ans les «agressifs-retirés» présentent moins de comportements prosociaux et sont plus rejetés par leurs pairs que les agressifs. Les travaux de Roll et Sells (1968 et 1972) ont par ailleurs montré que ce sont les 
enfants rejetés par leurs pairs qui sont les plus à risque de délinquance et autres inadaptations sociales à l'adolescence.

Utilisant cette approche rationnelle, nous avons fait l'hypothèse que les enfants «agressifs-retirés» seraient plus à risque d'inadaptation sociale «officielle» à l'adolescence que les enfants qui sont agressifs sans être retirés socialement, ou encore, plus à risque d'inadaptation que les enfants retirés sans être agressifs et, évidemment, plus à risque que les enfants ni agressifs ni retirés socialement.

\section{MÉTHODOLOGIE}

\section{ÉCHANTILLONNAGE}

Les sujets de la présente étude ont été choisis à l'origine pour une étude longitudinale de la schizophrénie (Ledingham, 1981; Schwartzman et al., 1985). Environ 4000 enfants de $1^{\text {re }}$, $4^{e}$ et $7^{e}$ années du primaire dans les écoles de milieux socioéconomiques moyens ou faibles [selon les indices de Forest (1972) et Crespo (1977)] de la Commission des écoles catholiques de Montréal (CECM), ont été évalués pour constituer les différents groupes de l'étude. Nous n'avons retenu, pour fin de prédiction de l'inadaptation à l'adolescence, que les sujets de première année du primaire. Au cours des premiers mois de 1977, 387 filles et 394 garçons ont été évalués par leurs pairs. À la même période en 1978, 168 filles et 164 garçons ont également été évalués par leurs pairs. Au cours de l'été 1985 nous avons consulté les dossiers des tribunaux de la jeunesse pour Montréal et sa région. Nous avons éliminé de l'échantillon tous les sujets repérés comme habitant à l'extérieur de Montréal et sa région. Le groupe de filles de la cohorte de 1977 est finalement constitué de 347 sujets, celui de la cohorte de 1978 est constitué de 146 sujets. Le groupe de garçons de la cohorte de 1977 est constitué de 349 sujets et celui de la cohorte de 1978 est constitué de 150 sujets.

\section{INSTRUMENTS}

Évaluation par les pairs : les enfants ont été évalués par leurs pairs à l'aide du Peer Evaluation Inventory de Pekarik 
et al. (1976). Cette évaluation permet d'obtenir des scores sur une échelle d'agressivité, une échelle de retrait social et une échelle de popularité (likeability). Les détails de la procédure sont décrits par Ledingham (1981).

Indices d'inadaptation sociale "officielle»; Les dossiers du Tribunal de la jeunesse de Montréal ainsi que ceux du Tribunal de la jeunesse de Longueuil ont été scrutés pour y retrouver les sujets de notre échantillon original qui auraient eu un dossier ouvert depuis la fin de leur première année scolaire (juillet 1977 ou 1978). Pour chaque dossier ouvert nous avons identifié sous quelle loi le dossier fut ouvert (Loi sur la protection de la jeunesse (LPJ), Loi sur les jeunes contrevenants (LJC, chapitre 48). Nous avons par la suite calculé un indice global (dossier ouvert) permettant de classifier les sujets selon qu'ils avaient ou non un dossier ouvert, pour quelque motif que ce soit au Tribunal de la jeunesse. Les sujets identifiés comme ayant un dossier sous la Loi sur les jeunes contrevenants recouvrent ceux qui étaient reconnus jeunes délinquants avant l'entrée en vigueur de la loi des jeunes contrevenants. Ces sujets peuvent donc être considérés des cas de délinquance juvénile «officiels». Les sujets identifiés comme ayant un dossier sous la Loi sur la protection de la jeunesse recouvrent ceux qui doivent être protégés de leur environnement (e.g. abus sexuel, violence physique) et ceux qui doivent être protégés parce qu'ils ont des «troubles de comportement». Nous n'avons pas distingué ces deux catégories parce que nous obtenons des nombres trop restreints pour effectuer des analyses statistiques. Étant donné le faible taux de base dans chacune des catégories de dossier, il nous semble utile de considérer que l'indice global (dossier ouvert) est un indice de difficulté d'adaptation sociale «officielle» et que pour le moment (i.e. en attendant une augmentation possible du taux de base avec l'avancement en âge des sujets) nous devons accepter cette limite pour effectuer une étude prédictive.

\section{TRAITEMENT STATISTIQUE}

Nous avons utilisé les deux indices (IOC et RIOC) mis au point par Loeber et Dishion (1983) pour vérifier la valeur de nos prédicteurs. Le IOC (Improvement Over Chance) est un calcul permettant de vérifier jusqu'à quel point l'utilisation d'un prédicteur permet d'améliorer la prédiction que nous aurions 
faite en distribuant les sujets au hasard parmi les quatre résultats possibles, i.e. sujets prédits délinquants et devenus délinquants (positifs valides), sujets prédits délinquants mais n'étant pas devenus délinquants (faux positifs), sujets prédits non délinquants et n'étant pas devenus délinquants (négatifs valides), sujets prédits non délinquants et devenus délinquants (faux négatifs). Le IOC, bien qu'il soit utile pour identifier jusqu'à quel point nous améliorons une prédiction qui serait faite au hasard, ne tient pas compte du succès maximum que l'on peut atteindre par notre prédiction. Le maximum de succès est dépendant du taux de délinquance dans la population étudiée (taux de base) et de la proportion d'individus qui sont identifiés à risque (ratio de sélection). Par exemple, si nous observons dans un échantillon que 12 sujets sur 146 sont effectivement devenus délinquants alors que notre critère de sélection identifiait 17 sujets sur les 146 comme étant à risque, notre taux de succès maximum est $96,58 \%$. Pour permettre une comparaison entre les différentes études utilisant des critères différents, Loeber et Dishion (1983) ont proposé d'utiliser ce "maximum prédictible» pour calculer un indice d'amélioration "relative» par rapport au hasard, i.e. le RIOC (Relative Improvement Over Chance). Il est à noter que, selon Loeber et Dishion, ces indices sont instables lorsque le taux de base est inférieur à $5 \%$. Les fréquences que nous avons obtenues sont généralement en deçà de ce seuil critique. Le lecteur devra tenir compte du fait qu'il s'agit de résultats qui devront être répliqués pour s'assurer de leur robustesse. Il faut cependant noter que nous travaillons sur deux cohortes pour chacun des sexes, nous obtenons ainsi une première réplique des résultats.

\section{RÉSULTATS}

\section{FREQUENCE DES DOSSIERS AU TRIBUNAL}

Au Tableau 1 nous présentons pour chaque groupe de sujets la fréquence et le pourcentage des sujets ayant et n'ayant pas de dossiers ouverts aux 4 catégories retenues: Loi sur les jeunes contrevenants, Loi de la protection de la jeunesse, chapitre 48 , l'un des trois précédents. 


\section{TABLEAU 1}

Fréquence et pourcentage des sujets des différents échantillons ayant un dossier au tribunal de la jeunesse

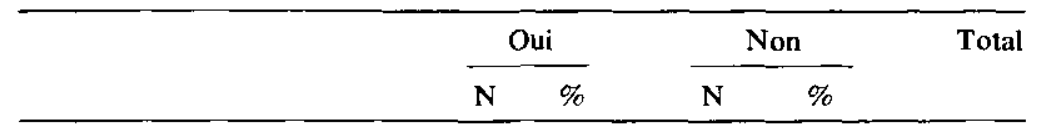

1. Loi sur les jeunes contrevenants

$\begin{array}{lllllcl}\text { Garçons } 1977 & 8 & 2,3 & 341 & 97,8 & 349 \\ \text { Garçons } & 1978 & 0 & 0 & 150 & 100 & 150 \\ \text { Filles } & 1977 & 1 & 0,3 & 346 & 99,7 & 347 \\ \text { Filles } & 1978 & 1 & 0,7 & 145 & 99,3 & 146\end{array}$

2. Loi sur la protection de la jeunesse

$\begin{array}{lrrrrrr}\text { Garçons } & 1977 & 10 & 2,9 & 339 & 97,1 & 349 \\ \text { Garçons } & 1978 & 3 & 2,0 & 147 & 98,0 & 150 \\ \text { Filles } & 1977 & 6 & 1,7 & 341 & 98,3 & 347 \\ \text { Filles } & 1978 & 4 & 2,7 & 142 & 97,3 & 146\end{array}$

3. Chapitre 48

$\begin{array}{lllllcl}\text { Garçons } 1977 & 2 & 0,6 & 347 & 99,4 & 349 \\ \text { Garçons } & 1978 & 0 & 0 & 150 & 100 & 150 \\ \text { Filles } & 1977 & 1 & 0,3 & 346 & 99,7 & 347 \\ \text { Filles } & 1978 & 0 & 0 & 146 & 100 & 146\end{array}$

4. Dossier ( 1 ou 2 ou 3 )

\begin{tabular}{|c|c|c|c|c|c|}
\hline \multirow[t]{2}{*}{$\begin{array}{l}\text { Garçons } 1 \\
\text { Garçons } 1 \\
\text { Filles } \\
\text { Filles }\end{array}$} & $\begin{array}{l}1977 \\
1978 \\
1977 \\
1978\end{array}$ & $\begin{array}{r}19 \\
3 \\
8 \\
5\end{array}$ & $\begin{array}{l}5,4 \\
2,0 \\
2,3 \\
3,4\end{array}$ & $\begin{array}{l}330 \\
147 \\
339 \\
141\end{array}$ & $\begin{array}{l}94,6 \\
98,0 \\
97,7 \\
96,6\end{array}$ \\
\hline & & 35 & 3,5 & 957 & 96,5 \\
\hline
\end{tabular}

Nous pouvons observer, qu'au total, 35 sujets $(3,5 \%)$ ont un dossier ouvert pour au moins un des motifs retenus. Les garçons les plus âgés (cohorte de 1977) ont plus de deux fois le nombre de dossiers ouverts $(5,4 \%)$ que les garçons de la cohorte de $1978(2 \%)$ et les filles de la cohorte de $1977(2,3 \%)$. Par contre, les filles de la cohorte de 1978 ont légèrement plus de dossiers ouverts $(3,4 \%)$ que les filles de la cohorte de $1977(2,3 \%)$.

Sauf pour la cohorte de garçons de 1977 , très peu de sujets ont un dossier ouvert sous la Loi sur les jeunes contrevenants et seulement 3 sujets pour l'ensemble de l'échantillon ont un dossier ouvert au titre du chapitre 48 . C'est sous la Loi sur la protection de la jeunesse que nous avons le plus de dossiers ouverts $(2,3 \%)$. Les garçons de la cohorte de 1977 et les filles de la cohorte de 1978 ont légèrement plus de dossiers ouverts à ce titre $(2,9 \%$ et $2,7 \%$ respectivement $)$ que les garçons de la cohorte $1978(2 \%)$ et les filles de la cohorte de $1978(1,7 \%)$. 


\section{PRÉDICTION DES DOSSIERS DU TRIBUNAL}

Étant donné les faibles fréquences de dossiers ouverts, nous ne présentons des résultats que pour l'ensemble des dossiers ouverts. Ces résultats sont présentés au tableau 2. Les sujets de chacune des cohortes ont été divisés selon les catégories de Ledingham (1981) : "agressif», "retiré», "agressifretiré», "contraste». La catégorie «autres» regroupe les sujets qui sont exclus des quatre catégories précédentes, i.e. ceux qui ont un score «agressivité» entre le $75^{\mathrm{e}}$ et le $95^{\mathrm{e}}$ percentile tout en ayant un score "retrait» inférieur au $75^{\mathrm{e}}$ percentile, et ceux qui ont un score "retrait» entre le $75^{\mathrm{e}}$ et le $95^{\mathrm{e}}$ percentile tout en ayant un score «agressivité» inférieur au $75^{\circ}$ percentile.

Nous pouvons observer au tableau 2 que la répartition des sujets, selon qu'ils ont ou n'ont pas un dossier ouvert au tribunal, correspond à peu près au hasard pour les deux cohortes de garçons, alors que pour les filles elle semble déterminée par la catégorie comportementale évaluée en première année du primaire.

Chez les garçons de 1976-1977, nous observons que le pourcentage des dossiers ouverts pour chaque catégorie de sujets correspond sensiblement au pourcentage de sujets dans cette catégorie : $63,2 \%$ vs $64,5 \%$ pour le groupe "contraste», $26,3 \%$ vs $21,8 \%$ pour le groupe «autres», $5,3 \%$ vs $7,7 \%$ pour le groupe «agressif-retiré», $5,3 \%$ vs $3,2 \%$ pour le groupe «agressif» et $0 \%$ vs $2,9 \%$ pour le groupe «retiré». Chez les garçons de 1977-1978, la répartition des dossiers ouverts correspond moins bien au hasard; les sujets des groupes «agressifsretirés» et "autres» sont surreprésentés $(33,3 \%$ vs $11,3 \%$ et $33,3 \%$ vs $21,8 \%$ ), alors que les sujets du groupe «autres» sont sous-représentés (33,3\% vs $63,3 \%$ ), mais le nombre de dossiers ouverts est faible (3) et le seuil de confiance déterminé par le chi-carré ne nous permet pas de rejeter l'hypothèse nulle.

Du côté des filles, le test statistique indique, pour les deux cohortes (1976-1977 et 1977-1978), que la répartition des sujets dévie sensiblement d'une répartition au hasard. Dans les deux cas ce sont les sujets classifiés "agressifs-retirés» en première année du primaire qui sont surreprésentés parmi ceux qui ont un dossier ouvert au tribunal. Chez les filles de la cohorte 19761977 , nous observons que $50 \%$ des dossiers ouverts se retrouvent parmi les filles classifiées «agressives-retirées» alors que ces 
filles ne représentent que $13 \%$ de cette cohorte. Cette surreprésentation correspond à la sous-représentation des sujets avec dossier ouvert pour la catégorie «contraste» $(12,5 \%$ vs $66,6 \%)$. Nous observons également une légère surreprésentation de dossiers ouverts à la catégorie «autres» $25 \%$ vs $15,3 \%$ ) et à la catégorie "agressif» $(12,5 \%$ vs $2,6 \%)$.

Chez les filles de la cohorte 1977-1978, nous observons que $60 \%$ des dossiers ouverts se retrouvent à la catégorie «agressifretiré", alors que cette catégorie représente uniquement $11,6 \%$ des sujets de cette cohorte. Cette surreprésentation est à peu de chose près tout à fait en rapport avec la sous-représentation de la catégorie «contraste» $(20 \%$ vs $70 \%)$.

Au tableau 3, est présentée une analyse de ces mêmes données selon la méthode proposée par Loeber et Dishion (1983) et décrite précédemment. Nous avons utilisé la catégorie «agressif» et «agressif-retiré» pour mettre en évidence la différence de résultats entre ces deux classifications. Dans les deux cas, les sujets classifiés dans la catégorie choisie sont comparés à tous les autres sujets. L'indice IOC permet de vérifier l'amélioration de la prédiction par rapport à une distribution faite au hasard et l'indice RIOC permet de vérifier cette amélioration de la prédiction en tenant compte du maximum atteignable.

Nous pouvons observer au tableau 3, que la classification «agressif» ne permet jamais une prédiction qui soit meilleure que celle faite au hasard, alors que la classification «agressifretiré» permet une amélioration par rapport au hasard pour les deux cohortes de filles. Dans le cas de la cohorte 1976-1977, l'amélioration «simple» par rapport au hasard (IOC) est de $1,71 \%$ et l'amélioration "relative au maximum prédictible» est de $42,54 \%$. Le succès de la prédiction est plus élevé pour la cohorte de 1977-1978 qui, rappelons-le, a un taux de base plus élevé. L'amélioration «simple» par rapport au hasard (IOC) est de $3,31 \%$ et l'amélioration relative est de $54,7 \%$. Ce succès de la prédiction pour les deux cohortes de filles se situe parmi les indices les plus élevẻs rapportés par Loeber et StouthamerLoeber (1985) pour la prédiction de la délinquance générale à l'adolescence par les comportements d'agression.

Nous ne présentons pas les résultats détaillés pour les sujets qui ont des dossiers ouverts sous la Loi sur les jeunes contrevenants, la Loi sur la protection de la jeunesse et le cha- 
pitre 48 parce que les taux de base sont trop faibles, mais il peut être utile, à titre indicatif, de décrire les grandes lignes de ces résultats. En ce qui concerne les 8 sujets de la cohorte des garçons de 1976-1977 qui ont un dossier ouvert sous la Loi sur les jeunes contrevenants, $50 \%$ (4) sont dans la catégorie «contraste», 37,5\% (3) sont dans la catégorie «autres» et 12,5\% (1) sont dans la catégorie «agressif-retiré». Aucun des sujets ne se retrouve dans la catégorie "agressif» ou «retiré». Cette distribution correspond à celle attendue par une distribution au hasard. Aucun garçon de la cohorte 1977-1978 n'a de dossier ouvert sous la LJC, alors qu'une fille de chaque cohorte a un dossier ouvert sous cette Loi. Dans un cas la fille classifiée au groupe «contraste» et dans le second au groupe «autres».

Pour la Loi sur la protection de la jeunesse, les résultats sont à peu de choses près identiques à ceux obtenus pour le regroupement de l'ensemble des dossiers ouverts. Ceci s'explique par le fait que la majorité des dossiers ouverts le sont sous la Loi de la protection de la jeunesse (voir tableau 1). Il est intéressant de noter que plus de la moitié des dossiers ouverts sous la LPJ faisaient référence à des «troubles du comportement» : $80 \%$ pour les garçons de $1976-1977,33 \%$ pour les garçons de 1977-1978, $83 \%$ pour les filles de 1976-1977 et $50 \%$ pour les filles de 1977-1978. Finalement, pour les 3 dossiers ouverts sous le chapitre 48, un garçon de 1976-1977 provenait du groupe «contraste», le deuxième provenait du groupe «autres» et la fille de 1976-1977 provenait du groupe «contraste».

\section{DISCUSSION}

Les résultats que nous venons de présenter indiquent clairement que les filles qui sont perçues par leurs pairs comme agressives et retirées socialement en première année du primaire sont plus à risque que les autres filles d'être référées au Tribunal de la jeunesse au cours des 7 ou 8 années qui vont suivre. Nous n'obtenons pas ce résultat pour les garçons. De fait, aucune des catégories du comportement utilisées ne permet de prédire les contacts des garçons avec le Tribunal de la jeunesse. Quelques hypothèses peuvent être avancées pour expliquer ces résultats, mais rappelons de nouveau que les taux de base de nos critères à prédire, i.e. les dossiers ouverts au tribunal, sont très faibles et que cette situation rend les résultats statistiques instables. 
La première hypothèse explicative de nos résultats a trait à la différence entre les dossiers ouverts sous la Loi sur les jeunes contrevenants et ceux ouverts sous la Loi sur la protection de la jeunesse. La majorité des dossiers ouverts pour les filles se réfèrent à la LPJ. Étant donné les caractéristiques des «agressifsretirés», i.e. des enfants rejetés, nous pourrions conclure que la catégorie «agressif-retiré» prédit les sujets qui auront besoin de protection parce qu'ils sont victimes de leur environnement, et ceci est plus fréquent chez les filles que chez les garçons. Une analyse des taux de base des dossiers ouverts sous la LPJ (voir tableau 1) nous permet de constater que ces taux de base sont équivalents entre les garçons et les filles, donc autant de garçons que de filles sont référés pour «protection» et, pourtant, nous ne prédisons de façon satisfaisante que les cas des filles. De plus, parmi les 13 filles pour lesquelles un dossier a été ouvert, seulement 3 ont été clairement référées parce qu'elles étaient «victimes» de leur environnement (un cas d'abus physique, un cas d'abus sexuel et un cas parce qu'il n'y a personne pour s'occuper d'elle). Les dix autres cas sont présentés comme ayant des troubles sérieux du comportement à l'adolescence, i.e. entre 13 ans et 15 ans.

La deuxième hypothèse explicative que nous pouvons invoquer fait référence aux différences de comportements entre les garçons et les filles au préscolaire et au début du primaire. Les comportements inadaptés, en général, et agressifs, en particulier, étant plus fréquents chez les garçons que chez les filles (Behar et Stringfield, 1974; McGee et al., 1985; Tremblay et Baillargeon, 1984), il est possible que les filles qui ont un comportement particulièrement aberrant soient plus faciles à identifier par leurs pairs. Pour caricaturer, nous pourrions dire qu'il est plus «anormal» pour une fille d'avoir des comportements aberrants et qu'elle est donc plus facile à repérer.

Cette dernière hypothèse mérite, à notre avis, d'être considérée sérieusement. Il est difficile présentement d'en apprécier la justesse parce que trop peu d'études prédictives de l'inadaptation des filles ont été réalisées. La majorité des efforts des chercheurs ont été centrés sur la prédiction de l'inadaptation des garçons, probablement parce qu'elle est plus abondante et irritante. Cependant, si l'inadaptation sociale des filles était plus facile à prédire et si elle a des conséquences plus néfastes sur la reproduction de l'inadaptation sociale d'une génération à l'autre 
(Castaignède et Tremblay, 1984; Favard, 1983: Robins et al., 1975) nous aurions identifié un moyen puissant d'aide à la prévention de la reproduction de l'inadaptation sociale.

En terminant, il importe de discuter notre incapacité de prédire la délinquance officielle (dossiers LJC). Les études prédictives antérieures utilisant l'évaluation par les pairs (e.g. Roff et Sells, 1968) nous permettaient de présumer que les cas de délinquance se retrouveraient parmi les individus identifiés les plus agressifs et plus particulièrement chez les agressifsrejetés, i.e. les agressifs-retirés dans notre classification. Le taux de base des cas de LJC chez les garçons de la cohorte de 1976-1977 était suffisamment élevé $(2,3 \%)$ pour que nous ayons une indication de la tendance de la répartition de ces cas parmi nos catégories. Il est clair que la moitié de ces garçons qui ont un dossier de délinquance officielle proviennent de la catégorie considérée la moins à risque, i.e. ceux qui, sur les échelles agressivité et retrait social, ont un score inférieur au $75^{\mathrm{e}}$ percentile (contraste). Un de ces garçons délinquants se retrouve dans le groupe «agressif retiré» et les 3 derniers se retrouvent dans la catégorie «autres». Si nous ne tenons compte que du score agressivité et que nous divisons les sujets selon qu'ils ont ou n'ont pas un score les plaçant au-dessus du $75^{\mathrm{e}}$ percentile, nous observons que 4 des 8 garçons ( $50 \%$ ) délinquants se retrouvent parmi les $25 \%$ plus agressifs. Ce résultat n'est cependant pas suffisant pour améliorer de façon significative la prédiction que nous aurions faite au hasard.

Nous constatons donc, en ce qui concerne les «tendances» de nos résultats pour les garçons, que la catégorie "agressif» utilisant le $75^{\mathrm{e}}$ percentile comme critère de dichotomie discrimine mieux les futurs délinquants que la catégorie «agressifretiré». Cependant, comme aucune catégorie n'atteint un seuil acceptable de probabilité, il faut conclure que l'évaluation du comportement par les pairs en première année du primaire ne permet pas, dans notre échantillon, de prédire la délinquance officielle des garçons avant l'âge de 16 ans.

Les sujets de cet échantillon seront suivis encore quelques années pour compléter l'étude de la prédiction de l'inadaptation sociale officielle de l'enfance à l'adolescence. Nous pouvons présumer que les taux de base augmenteront quelque peu et que nous serons en mesure de vérifier la stabilité des résultats 
obtenus dans la présente étude. Si les tendances observées dans la présente étude s'accentuent, il faudra conclure que la prédiction des cas de protection chez les filles doit être faite à l'aide de la classification "agressif-retiré» et les cas de délinquance chez les garçons à partir d'une simple évaluation de l'agressivité.

\section{RÉFÉRENCES}

BEHAR, L. et S. STRINGFIELD (1974), "A Behavior Rating Scale for the Preschool Child», Developmental Psychology, vol. 10 (5) : 601-610.

CASTAIGNÈDE, J. et R.E. TREMBLAY (1984), «Investissement parental et transmission de l'inadaptation sociale chez l'humain", dans A. de Haro et $\mathrm{X}$. Espadaler (édit.), Processus d'acquisition précoce. Les communications, Barcelone, Université autonome de Barcelone \& Société française pour l'étude du comportement animal.

CRAIG, M.M. et S.J. GLICK, «Ten Year Experience with the Glueck Social Prediction Table», Criminologica, $5:$ 17-27.

CRESPO, M. (1977), Un instrument pour le choix des écoles élémentaires dans le cadre de l'opération renouveau, Montréal, Commission des écoles catholiques de Montréal.

ERON, L.D. et al. (1971), Learning of Aggression in Children, Boston, Little, Brown \& Co.

FARRINGTON, D.P. (1979), «Environmental Stress, Delinquent Behavior, and Convictions", dans J.G. Sarason et C.D. Spielberger (édit.), Stress and Anxiety, vol. 6, Washington, Hemisphere.

FARRINGTON, D.P. (1983), «Delinquency from 10 to 25 », dans K.T. Van Dusen et S.A. Mednick (édit.), Antecedents of Aggression and Anti-Social Behavior, Boston, Kluwer-Nijhoff.

FAVARD, A.M. (1983), "La reproduction de l'inadaptation», Handicaps et inadaptations, 22 : 19-49.

FOREST, C. (1972), Handicaps sociopédagogiques de la clientèle de la C.E.C.M., Montréal, Commission des écoles catholiques de Montréal.

GLUECK, S. et E.T. GLUECK (1950), Unraveling Juvenile Delinquency, Cambridge, Mass., Harvard University Press.

GLUECK, S. et E.T. GLUECK (1972), Identification of Predelinquents, N.Y., Intercontinental Medical Book Corporation.

HUESMANN, L.R., L.D. ERON, M.M. LEFKOWITZ et L.O. WALDER (1984), "Stability of Aggression Over Time and Generations", Developmental Psychology, vol. 20 (6) : 1120-1134.

LEDINGHAM, J. (1981), "Developmental Patterns of Aggressive and Withdrawn Behavior in Childhood : a Possible Method for Identifying Preschizophrenics», Journal of Abnormal Child Psychology, 9: 1-22. 
LEFKOWTZ, M. et al. (1977), Growing up to be Violent : a Longitudinal Study of the Development of Aggression, Toronto, Pergamon Press.

LOEBER, R.E. et T. DISHION (1983), «Early Predictors of Male Delinquency, a Review», Psychological Bulletin 94 : 69-99.

LOEBER, R. et M. STOUTHAMER-LOEBER (1985), "The Prediction of Delinquency", dans H.C. Quay (édit.), Handbook of Juvenile Delinquency, New York, Wiley.

LYONS, J.A. (1984), Naturalistic Observation of Peer-Identified Agressive, Withdrawn, Aggressive-Withdrawn, and Contrast Children (Doctoral Thesis), Montréal, Concordia University, Department of Psychology.

MCGEE, R. et al. (1985), "The Rutter Scale for Completion by Teachers: Factor Structure and Relationships with Cognitive Abilities and Family Adversity for a Sample of New Zealand Children", Journal of Child Psychology and Psychiatry, 26, 727-739.

MILICH, R. et S. LANDAU (1984), «A Comparison of the Social Status and Social Behavior of Aggressive and Aggressive/Withdrawn Boys", Journal of Abnormal Child Psychology, 12 : 277-288.

PEKARIK, E.G. et al. (1976), «The Pupil Evaluation Inventory : a Sociometric Technique for Assessing Children's Social Behavior», Journal of Abnormal Child Psychology, 4 : 83-97.

ROBINS, L.N., P.A. WEST et B.L. HERJANIC (1975), "Arrest and Delinquency in Two Generations : a Study of Black Urban Families and their Children", Journal of Child Psychology and Psychiatry and Allied Disciplines, $16: 125-140$.

ROFF, M. et S.B. SELLS (1968), "Juvenile Delinquency in Relation to Peer Acceptance, Rejection and Socio-Economic Status», Psychology in the Schools, vol. $5: 3-18$.

ROFF, M., S. SELLS et M. GOLDEN (1972), Social Adjustment and Personality Development in Children, University of Minnesota Press, Minneapolis.

SCHWARTZMAN, A.E., J.E. LEDINGHAM et L.A. SERBIN (1985), «Identification of Children at Risk for Adult Schizophrenia : A Longitudinal Study", International Review of Applied Psychology, 34 : 363-380.

TREMBLAY, R.E. et L. BAILLARGEON (1984), «Les difficultés de comportement d'enfants immigrants dans les classes daccueil, au préscolaire", Revue canadienne de l'éducation, 9 (2) : 154-170.

WEST, D.J. (1982), Delinquency : Its Roots, Careers and Prospects, Cambridge, Harvard University Press.

WEST, D.J. et D.P. FARRINGTON (1973), Who Becomes Delinquent?, London, Heinemann.

WEST, D.J. et D.P. FARRINGTON (1977), The Delinquent Way of Life, N.Y., Crane Russak.

WOLFGANG, M.E., E.M. FIGLIO et T. SELLIN (1972), Delinquency in a Birth Cohort, Chicago, University of Chicago Press. 
TABLEAU 2

Comparaison des différents groupes de sujets pour la fréquence des dossiers ouverts au tribunal de la jeunesse

Dossier oui/non Agressif Agressif-retir

Retiré Contraste Autres

1. Garçons 1976-1977

$\begin{array}{lc}\text { Oui \% } & 5,3 \\ \text { (N) } & (1) \\ \text { Non } \% & 3,0 \\ \text { (N) } & (10) \\ \text { Total \% } & 3,2 \\ \text { (N) } & (11) \\ \mathbf{X}^{2}=1,21 & \mathrm{p}=\text { N.S }\end{array}$

$$
\mathrm{X}^{2}=1,21 \quad \mathrm{p}=\mathrm{N} . \mathrm{S}
$$

2. Garçons $1977-1978$

Oui \%
(N)
Non \%
(N)
Total \%
$(\mathrm{N})$
$\mathrm{X}^{2}=2,02$

3. Filles 1976-1977

$\begin{array}{lc}\text { Oui \% } & 12,5 \\ \text { (N) } & ,(1) \\ \text { Non.\% } & 2,4 \\ \text { (N) } & (8) \\ \text { Total \% } & 2,6 \\ \text { (N) } & (9) \\ \mathbf{X}^{2}=16,07 & \mathrm{p}<, 003\end{array}$

4. Filles $1977-1978$

\begin{tabular}{cccccc} 
Oui $\%$ & 0 & 60,0 & 0 & 20,0 & 20,0 \\
(N) & $(0)$ & $(3)$ & $(0)$ & $(1)$ & $(1)$ \\
Non $\%$ & 0,7 & 9,9 & 1,4 & 72,3 & 15,6 \\
Total $\%$ & 0,7 & 11,6 & 1,4 & 70,5 & 15,8 \\
$(\mathrm{~N})$ & $(1)$ & $(17)$ & $(2)$ & $(103)$ & $(23)$ \\
$\mathrm{X}^{2}=12,43$ & $\mathrm{p}<, 01$ & & & & \\
\hline
\end{tabular}

\section{5,3}

(1)

7,9

(26)

7,7

(27)

$\begin{array}{lll}0 & 33,3 & 33,3 \\ (0) & (1) & (1) \\ 2,7 & 63,9 & 21,8 \\ (4) & (94) & (32) \\ 2,7 & 63,3 & 22,0 \\ (4) & (95) & (33)\end{array}$

33,3

(1)

10,9

(16)

\section{0}

$63,2 \quad 26,3$

3,0

(10)

(12)

64, 515

(213) (71)

$\begin{array}{lll}2,9 & 64,5 & 21,8\end{array}$

(10) (225) (76)
11,3

(17)

0,7

$\mathrm{p}=\mathbf{N} . \mathbf{S}$.

50,0

(4)

12,1

(41)

13,0

(45)

0

12,5

25,0

(0)

(9)

2,6

(9)

(1)

67,8

(230)

(231) 15,3

(53) 


\section{TABLEAU 3}

Fréquence, pourcentage et indice d'amélioration de la prédiction par rapport au hasard (IOC et RIOC) pour le total des dossiers ouverts

$$
\text { (LPJ - LJC - chap. 48) }
$$

\begin{tabular}{|c|c|c|c|c|c|c|c|c|c|c|c|c|c|c|}
\hline \multirow{2}{*}{\multicolumn{4}{|c|}{ Prédicteur / année / sexe }} & \multicolumn{2}{|c|}{ Positifs valides } & \multicolumn{2}{|c|}{ Faux positifs } & \multicolumn{2}{|c|}{ Négatifs valides } & \multicolumn{2}{|c|}{ Faux négatifs } & \multirow{2}{*}{$p\left(x^{2}\right)$} & \multirow{2}{*}{$\frac{\text { IOC }}{\%}$} & \multirow{2}{*}{$\frac{\text { RIOC }}{\%}$} \\
\hline & & & & $\mathbf{N}$ & $\%$ & $\mathbf{N}$ & $\%$ & $\mathrm{~N}$ & $\%$ & $\mathbf{N}$ & $\%$ & & & \\
\hline Agressif & I & $\begin{array}{l}77 \\
78 \\
77 \\
78\end{array}$ & $\begin{array}{l}/ \mathrm{M} \\
\mathrm{M} \\
\mathrm{F} \\
\mathrm{F}\end{array}$ & $\begin{array}{l}1 \\
0 \\
1 \\
0\end{array}$ & $\begin{array}{c}9,1 \\
0 \\
11,1 \\
0\end{array}$ & $\begin{array}{r}10 \\
1 \\
8 \\
1\end{array}$ & $\begin{array}{c}90,9 \\
100 \\
88,9 \\
100\end{array}$ & $\begin{array}{l}320 \\
146 \\
331 \\
140\end{array}$ & $\begin{array}{l}94,7 \\
98,0 \\
97,9 \\
96,6\end{array}$ & $\begin{array}{r}18 \\
3 \\
7 \\
5\end{array}$ & $\begin{array}{l}5,3 \\
2,0 \\
2,1 \\
3,4\end{array}$ & $\begin{array}{l}\text { N.S. } \\
\text { N.S. } \\
\text { N.S. } \\
\text { N.S. }\end{array}$ & & \\
\hline $\begin{array}{l}\text { Agressif- } \\
\text { retiré }\end{array}$ & 1 & $\begin{array}{l}77 \\
78 \\
77 \\
78\end{array}$ & $\begin{array}{l}\mathrm{M} \\
\mathrm{M} \\
\mathrm{F}\end{array}$ & $\begin{array}{l}1 \\
1 \\
4 \\
3\end{array}$ & $\begin{array}{r}3,7 \\
5,9 \\
8,9 \\
17,6\end{array}$ & $\begin{array}{l}26 \\
16 \\
41 \\
14\end{array}$ & $\begin{array}{l}96,3 \\
94,1 \\
91,1 \\
82,4\end{array}$ & $\begin{array}{l}304 \\
131 \\
298 \\
127\end{array}$ & $\begin{array}{l}94,4 \\
98,5 \\
98,7 \\
98,4\end{array}$ & $\begin{array}{r}18 \\
2 \\
4 \\
2\end{array}$ & $\begin{array}{l}5,6 \\
1,5 \\
1,3 \\
1,6\end{array}$ & $\begin{array}{l}\text { N.S. } \\
\text { N.S. } \\
0,01 \\
0,01\end{array}$ & $\begin{array}{l}1,71 \\
3,31\end{array}$ & $\begin{array}{l}42,54 \\
54,71\end{array}$ \\
\hline
\end{tabular}

